\title{
Quality control of Photosystem II: reversible and irreversible protein aggregation decides the fate of Photosystem II under excessive illumination
}

\author{
Yasusi Yamamoto*, Haruka Hori, Suguru Kai, Tomomi Ishikawa, Atsuki Ohnishi, Nodoka Tsumura and \\ Noriko Morita
}

Graduate School of Natural Science and Technology, Okayama University, Okayama, Japan

\section{Edited by:}

Helmut Kirchhoff, Washington State University, USA

Reviewed by:

Dario Leister,

Ludwig-Maximilians-University

Munich, Germany

Rikard Fristedt, University of California

Los Angeles, USA

*Correspondence:

Yasusi Yamamoto, Graduate School of Natural Science and Technology,

Okayama University, 3-1-1

Tsushima-naka, Kita-ku,

Okayama 700-8530, Japan

e-mail: yasusiya@cc.okayama-u.ac.jp
In response to excessive light, the thylakoid membranes of higher plant chloroplasts show dynamic changes including the degradation and reassembly of proteins, a change in the distribution of proteins, and large-scale structural changes such as unstacking of the grana. Here, we examined the aggregation of light-harvesting chlorophyll-protein complexes and Photosystem II core subunits of spinach thylakoid membranes under light stress with 77K chlorophyll fluorescence; aggregation of these proteins was found to proceed with increasing light intensity. Measurement of changes in the fluidity of thylakoid membranes with fluorescence polarization of diphenylhexatriene showed that membrane fluidity increased at a light intensity of 500-1,000 $\mu \mathrm{mol}$ photons $\mathrm{m}^{-2} \mathrm{~s}^{-1}$, and decreased at very high light intensity $\left(1,500 \mu \mathrm{mol}\right.$ photons $\left.\mathrm{m}^{-2} \mathrm{~s}^{-1}\right)$. The aggregation of lightharvesting complexes at moderately high light intensity is known to be reversible, while that of Photosystem II core subunits at extremely high light intensity is irreversible. It is likely that the reversibility of protein aggregation is closely related to membrane fluidity: increases in fluidity should stimulate reversible protein aggregation, whereas irreversible protein aggregation might decrease membrane fluidity. When spinach leaves were preilluminated with moderately high light intensity, the qE component of non-photochemical quenching and the optimum quantum yield of Photosystem II increased, indicating that Photosystem II/light-harvesting complexes rearranged in the thylakoid membranes to optimize Photosystem II activity. Transmission electron microscopy revealed that the thylakoids underwent partial unstacking under these light stress conditions. Thus, protein aggregation is involved in thylakoid dynamics and regulates photochemical reactions, thereby deciding the fate of Photosystem II.

Keywords: Photosystem II, protein aggregation, non-photochemical quenching, photoinhibition, lipid peroxidation, membrane dynamics, thylakoid unstacking, quality control mechanism

\section{INTRODUCTION}

Photosynthesis has a well-known light intensity vs. activity profile (Taiz and Zeiger, 2006). Under low light, the rate of photosynthesis increases in proportion to light intensity, and the excitation energy captured by light-harvesting antennas is efficiently transferred to the reaction centers of Photosystems I (PSI) and II (PSII). As the light intensity increases, the rate of photosynthesis gradually decreases and reaches a plateau where no further increase in photosynthesis is seen. Although PSII can be photodamaged at this stage it is quickly repaired, so that the damage and repair of PSII are balanced. With increasing light intensity, PSII avoids stress by dissipating the excessive light energy as heat.

Energy-dependent quenching (qE; Birantais et al., 1980) is a major component of non-photochemical quenching (NPQ) of chlorophyll fluorescence (Genty etal., 1989), and is activated by acidification of the thylakoid lumen attained through $\mathrm{H}^{+}$uptake into the lumen coupled with electron transport (Birantais et al., 1980). This luminal acidification activates violaxanthin de-epoxidase (Yamamoto and Kamite, 1972), which causes de-epoxidation of the xanthophyll cycle carotenoid violaxanthin (Vio) to zeaxanthin (Zea) in light-harvesting complex (LHC) II (Demmig-Adams, 1990). The PsbS protein (Li et al., 2000), a transmembrane protein, is activated by the acidity of the thylakoid lumen and induces conformational changes and reversible aggregation of LHCII (Horton et al., 1996), although its exact location and function are not revealed yet. Thus, the aggregates of LHCII that are generated and stabilized by Zea are crucial for quenching excess energy and avoiding the risk of PSII over-excitation.

When the light intensity increases further, the maximum quantum efficiency of photosynthesis, as measured by chlorophyll fluorescence Fv/Fm, declines significantly. At this stage, photoinhibition prevails over protection and repair, and PSII is in a typical photodamaging state where degradation of the damaged D1 protein takes place (Barber and Andersson, 1992; Aro et al., 1993). Irreversible aggregation or cross-linking of the photodamaged reaction center-binding D1 protein also occurs (Mori and Yamamoto, 1992; Mori et al., 1995; Yamamoto and Akasaka, 1995; 
Ishikawa et al., 1999; Yamamoto, 2001; Yamamoto et al., 2008; Khatoon et al., 2009; Chan et al., 2012). The irreversible aggregation of the $\mathrm{D} 1$ protein is ascribed to the covalent cross-linking of the protein with nearby polypeptides after photooxidative damage to the D1 protein under light stress. Thus far, three cross-linked products of the D1 protein have been identified: the cross-linked products between D1 and D2 (Yamamoto et al., 2008), those between D1 and the $\alpha$-subunit of cytochrome $b_{559}$ (Barbato et al., 1995), and those between D1 and the core antenna chlorophyll binding protein CP43 (Yamamoto and Akasaka, 1995; Yamamoto et al., 2008). These cross-linked products are formed through photoinhibition of PSII via both donor-side and acceptor-side photoinhibition mechanisms, and are seen both in vitro and in vivo (Ohira et al., 2005). Once the cross-linked products are formed in the thylakoids, it is difficult to remove them by proteases, although some stromal protease(s) are able to degrade them (Ishikawa et al., 1999; Ferjani et al., 2001). Similar cross-linked products of the D1 protein were also observed in cyanobacterial thylakoids exposed to high light intensity (Lupinkova and Komenda, 2004). Thus, it is likely that cross-linking of the D1 protein with neighboring proteins is a general occurrence in oxygenic photosynthetic organisms subjected to excessive light.

Here, we carried out excessive illumination of spinach leaves or thylakoids and measured protein aggregation with 77K chlorophyll fluorescence, the $\mathrm{qE}$ of NPQ with pulse-amplitude-modulation (PAM) chlorophyll fluorometry, and membrane fluidity by fluorescence polarization of diphenylhexatriene (DPH). Samples were illuminated at various light intensities to more comprehensively understand the physiological meanings of protein aggregation under light stress. We also observed structural changes of thylakoids under light stress by TEM. These experiments enabled us to understand the nature of dynamic changes in the proteins, lipids, and thylakoid membranes under excessive illumination.

\section{MATERIALS AND METHODS \\ SAMPLE PREPARATION AND PRE-ILLUMINATION CONDITIONS}

Fresh spinach leaves were purchased from a local market in Okayama, Japan, and kept at $4^{\circ} \mathrm{C}$ in the dark room with a sufficient water supply to the roots before use. Intact thylakoid membranes were prepared as previously described (Yamamoto et al., 2004), with the omission of $\mathrm{Na}$-ascorbate from the grinding medium to avoid its effects on the observation of protein photodamage. The thylakoid membranes were suspended in a buffer solution containing $0.1 \mathrm{M}$ sorbitol, $15 \mathrm{mM} \mathrm{NaCl}, 5 \mathrm{mM} \mathrm{MgCl}_{2}, 30 \%$ (v/v) ethylene glycol, and $50 \mathrm{mM}$ Tricine- $\mathrm{KOH} \mathrm{pH} 7.5$ (solution A), frozen in liquid nitrogen and stored at $-80^{\circ} \mathrm{C}$ until use. For all experiments, samples were washed once and suspended in solution A without ethylene glycol (solution B). All procedures were carried out in darkness under a green safe light. Chlorophyll concentrations were determined using an $80 \%$ acetone extract and a U-3900 spectrophotometer (Hitachi, Tokyo, Japan).

Pre-illumination of spinach leaves was performed with white light from a tungsten-halogen light source (LA-180Me-R, Hayashi, Japan), which was filtered through a flat glass bottle containing $\mathrm{CuSO}_{4}$ solution to absorb heat from the light source.
Light intensity was measured with a LI-189 photometer (LICOR, Lincoln, NE, USA). For pre-illumination of the thylakoids, samples were adjusted to $0.1 \mathrm{mg}$ chlorophyll $\mathrm{mL}^{-1}$, placed in $0.5 \mathrm{~mL}$ transparent plastic tubes and incubated under illumination with white light and various light intensities in a thermostatic water bath at $20^{\circ} \mathrm{C}$. The pre-illumination was terminated by transferring the plastic tubes to an ice bath in the dark.

\section{MEASUREMENT OF CHLOROPHYLL PROTEIN AGGREGATION}

Aggregation of chlorophyll-binding proteins was examined by measuring chlorophyll fluorescence emission spectra at $77 \mathrm{~K}$. Spinach thylakoids were suspended in solution B at chlorophyll concentrations of $0.01 \mathrm{mg}$ chlorophyll $\mathrm{mL}^{-1}$ and frozen in liquid nitrogen. Fluorescence was measured with a fluorescence spectrophotometer (Jasco FP-8300, Japan) equipped with a low temperature unit. The excitation wavelength was $435 \mathrm{~nm}$ with a $20 \mathrm{~nm}$ band width, while the emission wavelength was 650-750 nm with a $2.5 \mathrm{~nm}$ band width. Curve fitting analysis was carried out with JASCO software Spectra Manager attached to the instrument. The six main components were identified according to Stoitchkova et al. (2006), and are referred to as F680 (peak, $681 \mathrm{~nm}$; half band width, $10.1 \mathrm{~nm}$ ), F685 (peak, $685 \mathrm{~nm}$; half band width, $9.3 \mathrm{~nm}$ ), F695 (peak, 693 nm; half band width, 9.2 nm), F700 (peak, $700 \mathrm{~nm}$; half band width, $15.8 \mathrm{~nm}$ ), F720 (peak $720 \mathrm{~nm}$; half band width, $21.9 \mathrm{~nm}$ ), and F735 (peak $735 \mathrm{~nm}$; half band width $23.4 \mathrm{~nm}$ ). They correspond to the fluorescence maxima of the trimeric and monomeric forms of LHCII, the PSII reaction center complex, the core antenna complex of PSII, the aggregated trimers of LHCII, the core complexes of PSI, and LHCI, respectively.

\section{ANALYSIS OF PSII ACTIVITY AND qE OF NPO}

Chlorophyll fluorescence parameters Fv/Fm, NPQ, and $\mathrm{qE}$ were measured with a Mini-PAM chlorophyll fluorometer (Walz, Effeltrich, Germany). Prior to the measurements, spinach leaves were kept at $4^{\circ} \mathrm{C}$ in the dark for $2 \mathrm{~h}$. For the Fv/Fm measurement, leaves were illuminated with white light of given light intensities for $1 \mathrm{~h}$ at various temperatures from 4 to $40^{\circ} \mathrm{C}$ (the light samples), and were then incubated in the dark for $10 \mathrm{~min}$ at $20^{\circ} \mathrm{C}$ before measurement. The dark control samples were incubated for $1 \mathrm{~h}$ in the dark. For the measurements of NPQ and $\mathrm{qE}$, leaves were illuminated at given light intensities at $20^{\circ} \mathrm{C}$ for $20 \mathrm{~min}$ (the light sample), while the dark samples were incubated in the dark at $20^{\circ} \mathrm{C}$ for $20 \mathrm{~min}$. The leaves were then incubated in the dark for 5 min and subjected to fluorescence measurement.

\section{MEASUREMENT OF MEMBRANE FLUIDITY}

Three micromolar DPH was added to the thylakoid suspension containing $0.01 \mathrm{mg}$ chlorophyll $\mathrm{mL}^{-1}$, and the mixture was incubated for $5 \mathrm{~min}$ at $20^{\circ} \mathrm{C}$ in the dark. Fluorescence polarization was measured with a JASCO fluorescence spectrophotometer (FP-8300) equipped with an automatic fluorescence polarization measuring unit (FDP-837) and a temperature controller (EHC813). The excitation and emission wavelengths were 360 and $430 \mathrm{~nm}$, respectively, and the band widths of excitation and emission were $5 \mathrm{~nm}$ and $20 \mathrm{~nm}$, respectively. The temperature was increased from 5 to $40^{\circ} \mathrm{C}$ at a rate of $5^{\circ} \mathrm{C} \mathrm{min}^{-1}$. 


\section{ELECTRON MICROSCOPY}

The spinach leaves were kept in the dark overnight at $15^{\circ} \mathrm{C}$ and used for the dark control samples. The light stressed samples were obtained from leaves illuminated for $1 \mathrm{~h}$ with white light (light intensity: $1,500 \mu \mathrm{mol}$ photons $\mathrm{m}^{-2} \mathrm{~s}^{-1}$ ) after adaptation to the dark. The dark and light-treated leaves were cut into $1 \times 3 \mathrm{~mm}$ fragments, pre-fixed in 3\% paraformaldehyde and 2.5\% glutaraldehyde, and post-fixed with $1 \% \mathrm{OsO}_{4}$. After embedding in plain resin, the samples were thin-sectioned into $70 \mathrm{~nm}$-thick samples with an ultramicrotome (Leica EM UC7, Germany). Electron micrographs were obtained with a transmission electron microscope (Hitachi H-7650).

\section{RESULTS}

\section{MEASUREMENT OF PROTEIN AGGREGATION BY 77K CHLOROPHYLL FLUORESCENCE}

The formation of LHCII aggregates under excessive illumination has previously been monitored by $77 \mathrm{~K}$ chlorophyll fluorescence emission spectra (Ruban and Horton, 1992; Stoitchkova et al., 2006; Haferkamp etal., 2010). We carried out the same measurement of $77 \mathrm{~K}$ chlorophyll emission spectra using spinach thylakoids pre-illuminated with excessive light of various intensities. Through a curve fitting analysis, we determined the amplitude of simple Gaussian curves representing fluorescence emission peaks of the aggregated trimers of LHCII at $700 \mathrm{~nm}$ (F700), and free trimeric and monomeric forms of LHCII at $680 \mathrm{~nm}$ (F680; Figure 1A). LHCII aggregation brought about an increase in the ratio of F700:F680 at 77K (Figure 1B). We also determined the ratios of F680, F685, F695, and F700 to F720 (Figure 1C), assuming that the level of F720 representing fluorescence from the core complex of PSI is constant over the light intensities examined. The increase in the ratio of F700:F680 as well as that of F700:F690 under excessive light conditions indicates an increase in LHCII aggregation, while the decrease in F680:F690, F685:F690, and F695:F690 suggests a decrease in the level of chlorophyll fluorescence caused by aggregation of related proteins.

\section{MEASUREMENT OF THYLAKOID MEMBRANE FLUIDITY}

We observed changes in the membrane fluidity of spinach thylakoids caused by illumination using DPH fluorescence polarization measurements. DPH is a popular fluorescence probe that associates with the hydrophobic region of membranes (Figure 2A). A detailed theoretical background of DPH usage has been given previously (Lentz, 1989; Franova et al., 2010). It is widely used to monitor membrane fluidity and the ordering of lipid acyl chains. We first measured changes in membrane fluidity according to the incubation temperature of the thylakoids (Figure 2B). As expected, decreases in $p$-values reflecting increases

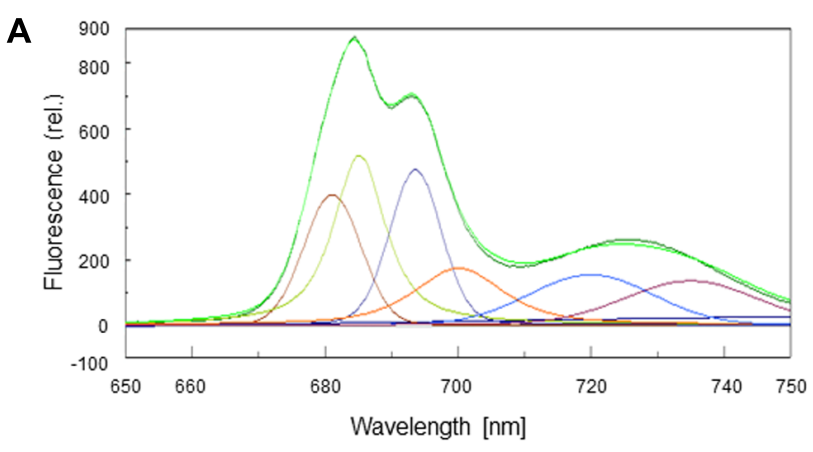

B

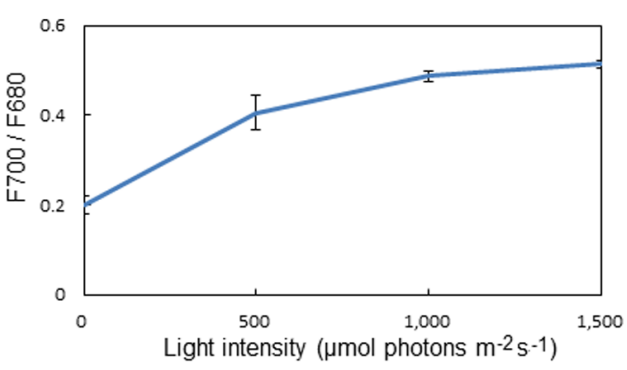

FIGURE 1 | Chlorophyll fluorescence emission spectra of spinach thylakoids at 77K. (A) A typical fluorescence emission spectrum with Gaussian decompositions from thylakoids kept in the dark. The six main components were identified as F680 (peak, 681 nm), F685 (peak, 685 nm), F695 (peak, 693 nm), F700 (peak, 700 nm), F720 (peak 720 nm), and F735 (peak $735 \mathrm{~nm}$ ). They correspond to the fluorescence maxima of the trimeric and monomeric forms of LHCII, the PSII reaction center complex, the core antenna complex of PSII, the aggregated trimers of LHCII, the core complex of PSI, and $\mathrm{LHCl}$, respectively. (B) The ratio of $\mathrm{F} 700$ :F680 at various light
C

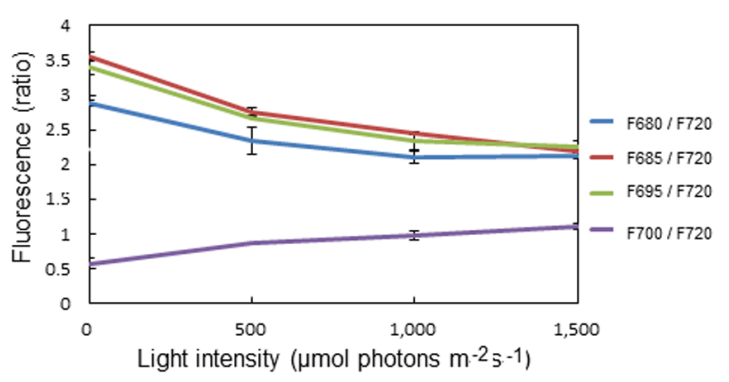

intensities. The increase in the ratio under excessive light indicates the conversion of free LHClls to aggregated ones. The data are the means of three independent measurements \pm S.D. (C) The ratios of F680:F720 (blue), F685:F720 (red), F695:F720 (green), and F700:F720 (purple) at various light intensities. The light-induced decrease in F680:F720 and increase in F700:F720 indicate LHCIl aggregation, while decrease in F685:F720 and F695:F720 suggests aggregation between the D1 protein and CP43. We assumed that the level of F720 is constant at various light intensities. The data are the means of three independent measurements \pm S.D. 


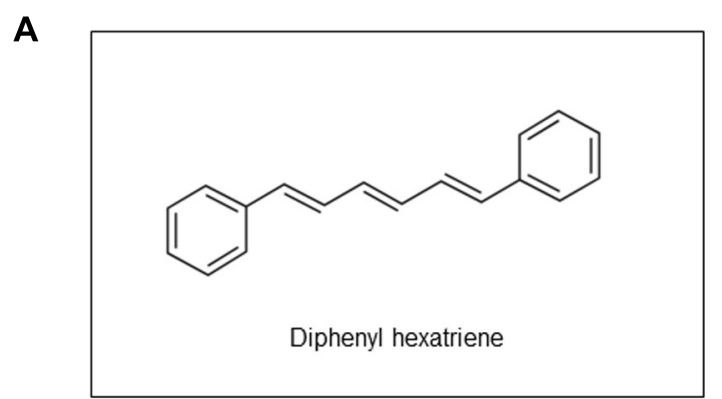

B

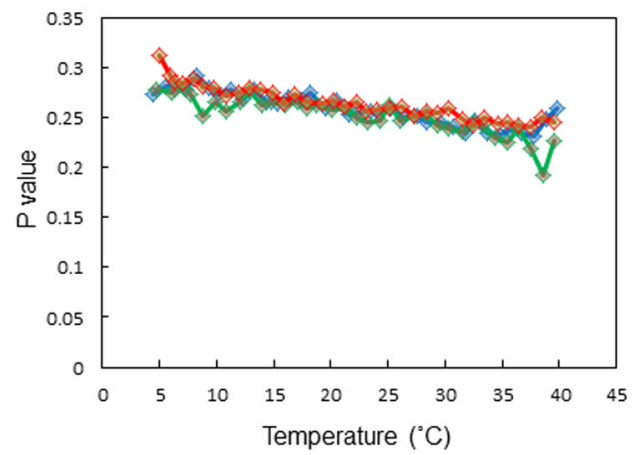

FIGURE 2 | Assay of the membrane fluidity of spinach thylakoids with fluorescence polarization of diphenylhexatriene (DPH). (A) The chemical structure of DPH. (B) The p-values of DPH fluorescence polarization in thylakoids incubated at different temperatures from 5 to $40^{\circ} \mathrm{C}$. Three different colors indicate three independent measurements.

\section{C}

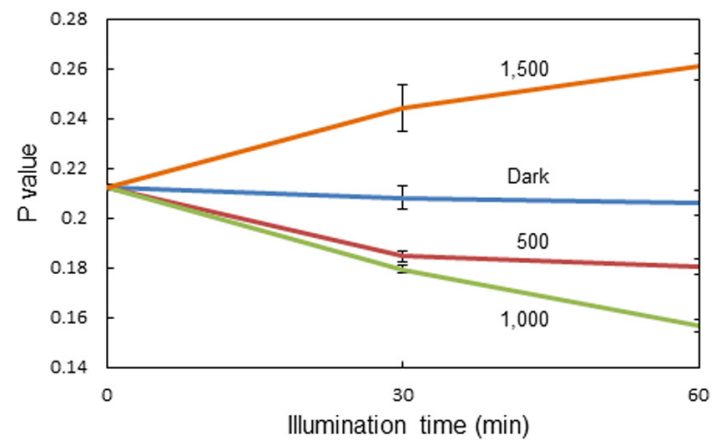

The increase and decrease in the $p$-value indicate a decrease and increase in membrane fluidity, respectively. (C) The $p$-values of DPH fluorescence polarization in thylakoids illuminated with white light of different intensities at $20^{\circ} \mathrm{C}$. The data are the means of three independent measurements \pm S.D. in membrane fluidity were observed when the incubation temperature was increased from 5 to $40^{\circ} \mathrm{C}$. Next, we examined the effects of excessive light on the $p$-value (Figure 2C). We detected a decrease in $p$-value, representing an increase in membrane fluidity at moderately high light conditions (light intensity: 500-1,000 $\mu \mathrm{mol}$ photons $\mathrm{m}^{-2} \mathrm{~s}^{-1}$ ), while the $p$-value increased at extremely high light conditions (light intensity: 1,500 $\mu \mathrm{mol}$ photons $\mathrm{m}^{-2} \mathrm{~s}^{-1}$ ) reflecting a decrease in membrane fluidity.

\section{MEASUREMENTS OF Fv/Fm, NPQ, AND qE}

We observed an increase in the optimum quantum yield of PSII, as measured by chlorophyll fluorescence Fv/Fm, in the spinach leaves that were pre-illuminated with moderately high light for a short period (Figures 3A,B). This was not seen at $4^{\circ} \mathrm{C}$. Pre-illumination at $20^{\circ} \mathrm{C}$ also induced an increase in $\mathrm{qE}$ in preilluminated leaves compared with dark-adapted leaves (Figures 3A,C). These data are consistent with the view that moderate light stress causes mobilization of PSII and LHCII complexes in the grana, thereby maintaining or increasing the efficiency of the PSII reaction. By contrast, prolonged illumination with higher light intensities decreased Fv/Fm as a result of photoinhibition.

A decrease in the chlorophyll fluorescence Fv/Fm always accompanies the photoinhibition of PSII. The decrease in the chlorophyll fluorescence yield may partially be caused by aggregation of the reaction-center binding proteins and the core antenna chlorophyll binding proteins induced by excessive light. In the curve analysis of $77 \mathrm{~K}$ chlorophyll fluorescence emission spectra, we observed a decrease in fluorescence components emitting at $685 \mathrm{~nm}$ (F685) and $695 \mathrm{~nm}$ (F695), which is attributable to the fluorescence from the reaction center complex and the core antenna complexes of PSII, respectively (Stoitchkova et al., 2006; Figure 1). The aggregation of D1 and CP43, demonstrated previously by western blot analysis to occur under severe light stress (Yamamoto et al., 2008), may be involved in the decrease in F685 and F695.

\section{LARGE-SCALE STRUCTURAL CHANGES OF THYLAKOIDS UNDER LIGHT STRESS}

Structural changes of thylakoids occur not only at a molecular level but also on a larger scale. We previously observed strong lightinduced unstacking of the grana in vitro using isolated spinach thylakoids (Khatoon et al., 2009), where membrane stacking and unstacking were estimated by measuring the chlorophyll content in heavy fractions representing the grana, after fractionation of the grana and stroma thylakoids by digitonin treatment and low speed centrifugation (Chow et al., 1980). Digitonin is a non-ionic detergent effective in separating the grana from the stroma thylakoids. Using this method, we observed that strong illumination induces a decrease in the grana fraction, so concluded that the grana show unstacking under light stress (Figure 4A). However, there may be other possible explanations. Light-induced shrinkage of the grana should also decrease the amount of pellet that results from centrifugation of digitonin-treated thylakoids. Light-induced bending 


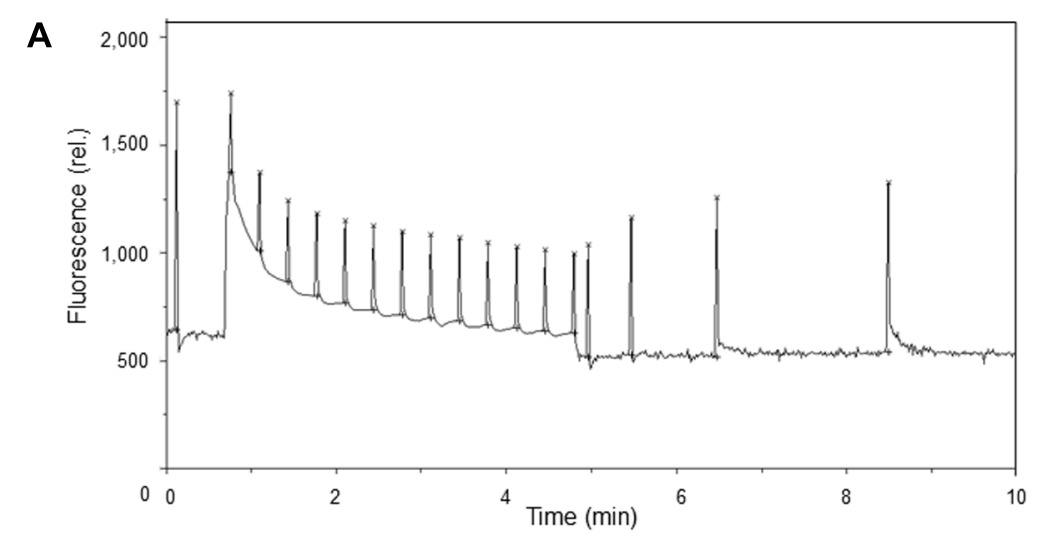

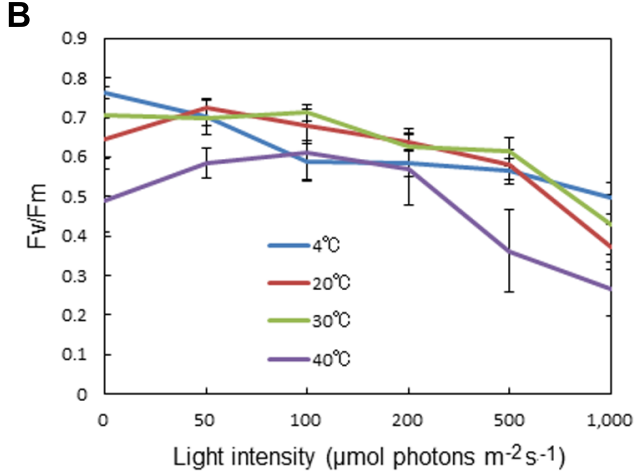

FIGURE 3 | Effects of pre-illumination of spinach leaves on chlorophyll fluorescence parameters Fv/Fm, NPQ, and qE. These parameters were measured with a Mini-PAM chlorophyll fluorometer (Walz, Germany). (A) A typical time course of fluorescence measurement for NPQ and $q E$. The light intensity of the continuous illumination was $600 \mu \mathrm{mol}$ photons $\mathrm{m}^{-2} \mathrm{~s}^{-1}$, and during the illumination several saturating flashes were given to estimate the formation of $\mathrm{qE}$. After continuous illumination, another series of

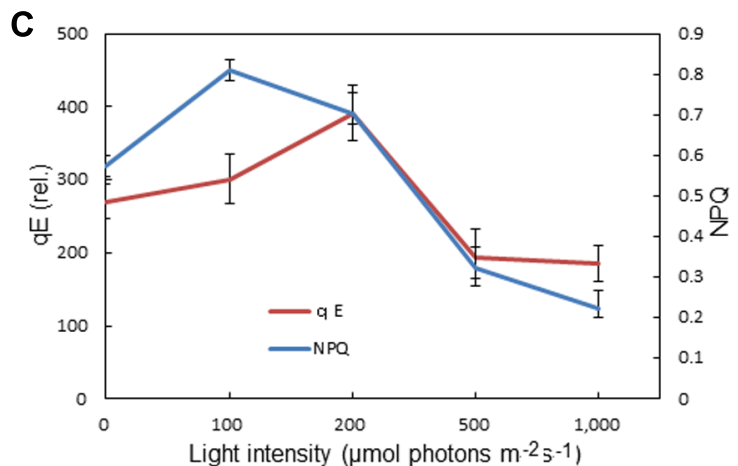

saturating flashes was given to the sample and the fluorescence intensity induced by the flash at the 4 min point in the dark period was used to calculate $\mathrm{qE}$. (B) The effects of pre-illumination of spinach leaves at various temperatures on $\mathrm{Fv} / \mathrm{Fm}$. The data are the means of three independent measurements \pm S.D. (C) The effects of pre-illumination of spinach leaves at $20{ }^{\circ} \mathrm{C}$ on NPQ and $q E$. The data are the means of three independent measurements \pm S.D. of the stromal thylakoids outward, corresponding to a partial unstacking of the thylakoids, may result in an increase in grana margins and a decrease in the grana core.

To more directly observe structural changes in the grana under light stress, we examined spinach thylakoid membranes using TEM (Figure 4B). As the chemical fixation of isolated spinach thylakoids may cause various artifacts, we used spinach leaves instead. Outward-bending of the stromal thylakoids was observed after strong illumination (light intensity: 1,500 $\mu$ mol photons $\mathrm{m}^{-2}$ $\mathrm{s}^{-1}$ ) of the spinach leaves for $1 \mathrm{~h}$. Accordingly, the size of the grana core appeared to be reduced after strong illumination.

\section{DISCUSSION}

\section{PROTEIN AGGREGATION}

Among the components that emit chlorophyll fluorescence at 77K, F700 was shown to be directly related to LHCII aggregation. The behavior of $\mathrm{F} 700$ in response to the acidic luminal $\mathrm{pH}$ and addition of antimycin $\mathrm{A}$, which inhibits $\mathrm{qE}$, showed that $\mathrm{qE}$ is stimulated or inhibited by the enhancement or disruption of LHCII aggregation, respectively (Horton et al., 1991). We detected an increase in F700 under illumination of the thylakoids with excessive light over a wide range of light intensities from 500 to $1,500 \mu$ mol photons $\mathrm{m}^{-2} \mathrm{~s}^{-1}$ (Figure 1). It is interesting to note that F680, 685, and 695 decreased with increased light intensity, confirming that the reaction center binding D1 protein and core antenna chlorophyll binding protein $\mathrm{CP} 43$ form irreversible aggregates under strong illumination (Yamamoto, 2001; Yamamoto et al., 2008).

In the measurement of reversible aggregation of LHCII by linear and circular dichroism spectroscopy, changes in the conformation of chlorophylls $a$ and $b$, and xanthophyll molecules were suggested (Ruban etal., 1997). It is likely that the Zea molecules in aggregated LHCII adopt a particular orientation suitable for the dissipation of excessive excitation. However, we do not know whether this stabilizes the aggregated LHCII or directly participates in the quenching process. It was suggested that Zea directly quenches the excessive singlet excitation energy of chlorophyll $a$ (Frank etal., 2000), while the results obtained by an in vitro study suggest that $\mathrm{qE}$ is generated when Zea induces LHCII aggregation (Gruszecki et al., 2006). Recent reconstitution experiments with liposomes containing LHCII, Zea, and PsbS support a positive role for all these components in $\mathrm{qE}$ (Wilk etal., 2013). Although LHCP aggregation in the $\mathrm{qE}$ mechanism is well documented through extensive studies, the exact physiological meanings of LHCII aggregation 


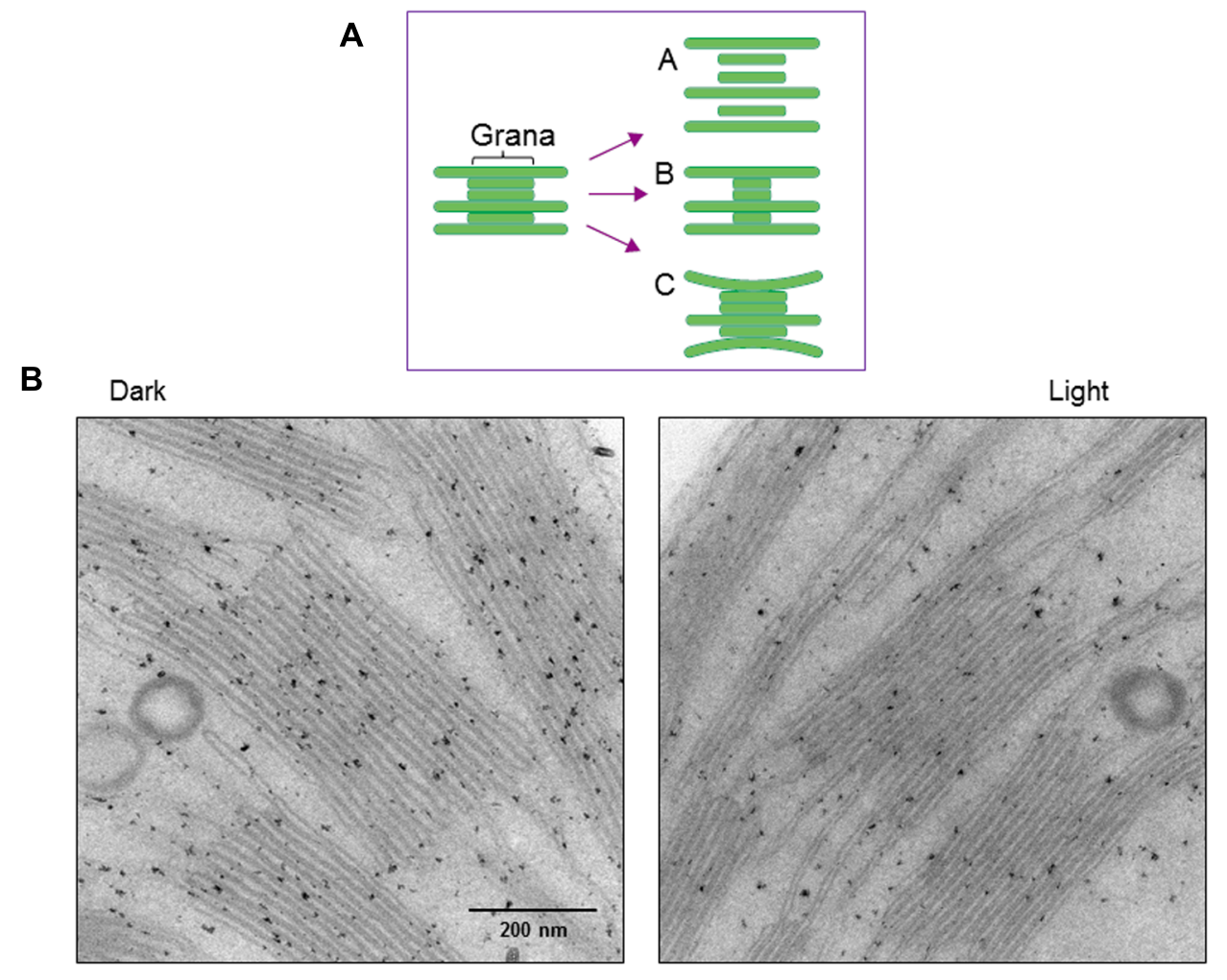

FIGURE 4 | Changes in thylakoid stacking in the chloroplasts after strong illumination of spinach leaves. (A) A diagram showing possible structural changes in the grana after illumination. Models $A$, $\mathrm{B}$ and $\mathrm{C}$ correspond to light-induced unstacking of the grana, shrinkage of the grana and bending of the stromal thylakoids outward. (B) Electron micrographs obtained by TEM. Model C in (A), which shows partial thylakoid unstacking, fits best with the present results. are still not completely clear (Duffy et al., 2013). By contrast, the mechanism of formation and physiological meanings of irreversible protein aggregation under severe light stress have been more clearly elucidated (Yamamoto, 2001; Yamamoto et al., 2008).

\section{MOLECULAR CROWDING IN THE THYLAKOID MEMBRANES}

The thylakoid membranes are crowded with proteins and protein complexes, such that lateral diffusion of these components is considerably restricted (Kirchhoff et al., 2008a,b). The crowded conditions are most typically seen in the grana regions of higher plant chloroplasts where the PSII complexes are enriched and sometimes show highly packed semi-crystalline arrays (Johnson et al., 2011). The thylakoids have to respond to incident light to efficiently capture light energy when the light is relatively weak and behave appropriately when the light is excessive. Thus, a suitable "crowd control mechanism" must work to optimize the photochemical reactions. The most important process in this is likely to be regulation of the lateral diffusion of PSII/LHCII supercomplexes and other related components on the thylakoids under fluctuating light conditions in the natural environment. In the grana thylakoids, overcrowded PSII/LHCII complexes may cause unnecessary overlap or aggregation of antenna complexes, thereby decreasing the efficiency of excitation energy trapping. Overcrowding also prevents the smooth movement of protein molecules on the membranes. By contrast, dilution of the PSII/LHCII supercomplexes in the thylakoids was shown to induce partial dissociation of minor LHCII complexes from the supercomplexes, leading to inefficient excitation trapping and transfer (Haferkamp et al., 2010). These data demonstrated that a suitable packing density of the PSII, as is realized in the natural grana, is important for efficient energy transfer from the antenna to the reaction center.

\section{MOBILE LIPIDS IN THE THYLAKOIDS AND CHANGES IN THE ORGANIZATION OF PHOTOSYSTEM II AND LHCII UPON EXCESSIVE ILLUMINATION}

Lipids are known to change the degree of saturation of constituent fatty acids, depending on the ambient temperature. This enables intrinsic proteins and lipid-soluble plastoquinones in the thylakoids to move over a wide range of temperatures. Fatty acid desaturases change saturated fatty acids to unsaturated ones when plants are exposed to lower temperatures, while saturases, which catalyze the reverse reaction, have not yet been identified. Polyunsaturated fatty acids become a target for lipid peroxidation, which may cause damage to proteins and induce irreversible aggregation or the cross-linking of proteins. Cross-linking is also expected with thylakoid lipids, which may affect the fluidity of thylakoid membranes, and therefore mobility of the membrane components. 
As membrane fluidity determines the lateral diffusion of lipids and proteins on the thylakoid membranes, the measurement of fluidity is an important but difficult task. Thus far, three methods have been successfully employed for the study of thylakoid membrane fluidity: fluorescence recovery after photobleaching (FRAP; Sarcina et al., 2003, 2006; Goral et al., 2010, 2011; Johnson etal., 2011; Herbstova etal., 2012; Kirchhoff et al., 2013), electron paramagnetic resonance (Tardy and Havaux, 1997; Kota et al., 2002), and fluorescence polarization measurement with a suitable fluorescence probe such as DPH (Yamamoto et al., 1981; Ford and Barber, 1983). Following FRAP measurements, cyanobacterial thylakoids and chloroplast grana thylakoids were shown to be relatively immobile, being limited in the lateral movement of supermolecular complexes (Sarcina et al., 2003; Herbstova et al., 2012). In particular the grana are highly crowded with PSII/LHCII complexes and it is apparently not easy for the PSII/LHCII supermolecular complexes to move around freely in these areas (Kirchhoff et al., 2008a). Indeed, only a small fraction of the membrane areas in the grana is mobile and the diffusion constants of the molecules in the mobile areas were experimentally determined to be small. Importantly, strong illumination increased membrane fluidity in cyanobacterial thylakoids (Sarcina et al., 2006) and in the grana of higher plant chloroplasts (Herbstova etal., 2012), thereby inducing mobilization of the PSII complexes.

These and other related studies have opened a new research area investigating light stress in terms of changes in the molecular arrangement of thylakoids, where the roles of LHCII aggregation in the $\mathrm{qE}$ process of NPQ and D1 protein and CP43 aggregation in photoinhibition are reevaluated. It should be noted here that all the methods described above to measure membrane fluidity are useful to monitor overall fluidity change in thylakoid membranes. However, they are not suitable to monitor specific changes in local membrane fluidity, in particular around the PSII/LHCII supercomplexes, under light stress. In spite of these difficulties, we monitored changes in thylakoid fluidity with the fluorescence polarization of DPH. We detected an increase in membrane fluidity under moderate light stress and a decrease under severe light stress (Figure 2).

The molecular mechanisms of increased membrane fluidity under moderate light stress are not fully understood, but there are several possibilities. Protein phosphorylation is known to be a driving force for the lateral movement of LHCII in thylakoid membranes (Allen, 1992, 2003). Under moderately high light conditions, light-induced phosphorylation of proteins takes place, which drives the lateral diffusion of PSII and LHCII complexes in the grana. This idea is supported by the study of thylakoid kinase mutants by Bonardi et al. (2005), in which no structural alteration occurred in the thylakoid kinase mutants $\sin 8$ or the double mutant $\operatorname{stn} 7 / 8$ of Arabidopsis thaliana (Herbstova etal., 2012). Many proteins are phosphorylated in the thylakoid membranes depending on different environmental conditions (Vener, 2007). Previously, degradation and aggregation of the D1 protein in spinach grana thylakoids under moderate heat stress were shown to be affected by protein phosphorylation and dephosphorylation (Komayama et al., 2007). Subsequently, this was studied more extensively using $\operatorname{stn} 7, \sin 8$, and $\operatorname{stn} 7 / 8$ A. thaliana mutants under high light stress, when it was demonstrated that not only degradation and aggregation of the D1 protein but also the overall structure of the thylakoid membranes is regulated by the reversible phosphorylation of thylakoid proteins (Fristedt et al., 2009). Thus, it is likely that protein phosphorylation plays an important role in the dynamics of thylakoids in higher plant chloroplasts. Indeed, mobilization of thylakoid proteins under moderate light stress increased $\mathrm{qE}$ of NPQ and even transiently activated PSII in the present study (Figure 3).

\section{STRUCTURAL CHANGES OF THYLAKOIDS}

Structural changes of the thylakoids under light stress are important in the quality control of PSII. We observed outward bending of the thylakoids at the margins of the grana under relatively strong illumination (Figure 4). The mechanism of the partial unstacking of the thylakoids remains to be determined, although, as described above, protein phosphorylation and dephosphorylation may be involved in this process. Recently, CURVATURE THYLAKOID 1 (CURT1) proteins responsible for the induction of membrane curvature were identified in A. thaliana (Armbruster et al., 2013), and it would be interesting to determine the effect of light stress on their function. These proteins are enriched at grana margins and are suggested to modify the thylakoid architecture, including grana stacking. Rearrangement of protein complexes in the grana may play crucial roles in grana stacking/unstacking.

We postulate that the outward bending and partial unstacking of the thylakoids can be physiologically explained as follows. First, the outward bending and partial unstacking of the grana would increase the area of the grana margins, which would aid degradation of photo-damaged D1 protein by specific protease(s). FtsH proteases are one candidate for the removal of photodamaged and heat-damaged D1 protein in the thylakoid (Kamata et al., 2005; Komenda et al., 2006; Yoshioka et al., 2006), and the grana margins are the sites of $\mathrm{FtsH}$ protease assembly prior to hexameric protease activation and reaction with damaged D1 protein in PSII that has moved from the grana core (Yoshioka et al., 2010). Increase in the area of the grana margins is necessary for the swift repair of damaged D1 proteins. Second, partial unstacking may prevent the production of ROS in the grana by stimulating the free movement of PSII and LHCII complexes. The mobilization of PSII and LHCII complexes should prevent the irreversible aggregation of the complexes and production of ROS from the impaired PSII in the aggregates. Indeed, previous work showed that thylakoid stacking artificially induced by the addition of $\mathrm{MgCl}_{2}$ stimulates production of hydroxyl radicals under light stress (Khatoon et al., 2009). Thus, the partial membrane unstacking observed under light stress appears to be a dynamic way of controlling the quality of PSII.

\section{CONCLUSION}

Reversible and irreversible aggregation of LHCII and PSII complexes under moderate and strong light stresses appear to be closely related to the $\mathrm{qE}$ component of NPQ and the photoinhibition of PSII, respectively. Although protein aggregation is a subtle phenomenon observed under light stress, it plays a crucial role in the quality control of PSII. It may also be related to the 
microscopic rearrangement of PSII/LHCII super complexes in the thylakoids and to thylakoid unstacking under light stress, although further studies are required to fully understand the details of these processes.

\section{AUTHOR CONTRIBUTIONS}

Yasusi Yamamoto organized and wrote the manuscript. Haruka Hori, Suguru Kai, Tomomi Ishikawa, Atsuki Ohnishi, and Nodoka Tsumura carried out the experiments and obtained the data presented here. Noriko Morita organized the data and prepared the figures.

\section{ACKNOWLEDGMENTS}

This work was supported by Grants-in-aid for Scientific Research 24570053 from the Ministry of Education, Culture, Sports, Science and Technology of Japan to Yasusi Yamamoto. The authors thank Dr. Miho Nishimura and Mr. Daisuke Nanba for technical advice on TEM.

\section{REFERENCES}

Allen, J. F. (1992). How does protein phosphorylation regulate photosynthesis? Trends Biochem. Sci. 17, 12-17. doi: 10.1016/0968-0004(92)90418-9

Allen, J. F. (2003). State transitions - a question of balance. Science 299, 1530-1532. doi: 10.1126/science. 1082833

Armbruster, U., Labs, M., Pribil, M., Viola, S., Xu, W., Scharfenberg, M., et al. (2013). Arabidopsis CURVATURE THYLAKOID1 proteins modify thylakoid architecture by inducing membrane curvature. Plant Cell 25, 2661-2678. doi: 10.1105/tpc.113.113118

Aro, E. M., Virgin, I., and Andersson, B. (1993). Photoinhibition of Photosystem II. Inactivation, protein damage and turnover. Biochim. Biophys. Acta 1143, 113-134. doi: 10.1016/0005-2728(93)90134-2

Barbato, R., Friso, G., Ponticos, M., and Barber, J. (1995). Characterization of the light-induced cross-linking of the alpha-subunit of cytochrome b559 and the D1 protein in isolated photosystem II reaction centers. J. Biol. Chem. 270, 24032-24037. doi: 10.1074/jbc.270.41.24032

Barber, J., and Andersson, B. (1992). Too much of a good thing: light can be bad for photosynthesis. Trends Biochem. Sci. 17, 61-66. doi: 10.1016/09680004(92)90503-2

Birantais, J.-M., Vernotte, C., Picaud, M., and Krause, G. H. (1980). Chlorophyll fluorescence as a probe for the determination of the photo-induced proton gradient in isolated chloroplasts. Biochim. Biophys. Acta 591, 198-202. doi: 10.1016/0005-2728(80)90233-9

Bonardi, V., Pesaresi, P., Becker, T., Schleiff, E., Wagner, R., Pfannschmidt, T., et al. (2005). Photosystem II core phosphorylation and photosynthetic acclimation require two different protein kinases. Nature 437, 1179-1182. doi: 10.1038/nature04016

Chan, T., Shimizu, Y., Pospisil, P., Nijo, N., Fujiwara, A., Taninaka, Y., et al. (2012). Quality control of photosystem II: lipid peroxidation accelerates photoinhibition under excessive illumination. PLoS ONE 7:e52100. doi: 10.1371/journal.pone.0052100

Chow, W. S., Thorne, S. W., Duniec, J. T., Sculley, M. J., and Boardman, N. K. (1980). The stacking of chloroplast thylakoids. Effects of cation screening and binding, studied by the digitonin method. Arch. Biochem. Biophys. 201, 347-355. doi: 10.1016/0003-9861(80)90520-2

Demmig-Adams, B. (1990). Carotenoids and photoprotection in plants: a role for the xanthophyll zeaxanthin. Biochim. Biophys. Acta 1020, 1-24. doi: 10.1016/0005-2728(90)90088-L

Duffy, C. D., Valkunas, L., and Ruban, A. V. (2013). Light-harvesting processes in the dynamic photosynthetic antenna. Phys. Chem. Chem. Phys. 15, 18752-18770. doi: 10.1039/C3CP51878G

Ferjani, A., Abe, S., Ishikawa, Y., Henmi, T., Yuka, T., Nishi, Y., et al. (2001). Characterization of the stromal protease(s) degrading the crosslinked products of the D1 protein generated by photoinhibition of photosystem II. Biochim. Biophys. Acta 1503, 385-395. doi: 10.1016/S0005-2728(00) 00233-4
Ford, R. C., and Barber, J. (1983). Time-dependent decay and anisotropy of fluorescence from diphenylhexatriene embedded in the chloroplast thylakoid membrane. Biochim. Biophys. Acta 722, 341-348. doi: 10.1016/0005-2728(83) 90082-8

Frank, H. A., Bautista, J. A., Josue, J. S., and Young, A. J. (2000). Mechanism of nonphotochemical quenching in green plants: energies of the lowest excited singlet states of violaxanthin and zeaxanthin. Biochemistry 39, 2831-2837. doi: 10.1021/bi9924664

Franova, M., Repakova, J., Capkova, P., Holopainen, J. M., and Vattulainen, I. (2010). Effects of DPH on DPPC-cholesterol membranes with varying concentrations of cholesterol: from local perturbations to limitations in fluorescence anisotropy experiments. J. Phys. Chem. B 114, 2704-2711. doi: 10.1021/jp908533x

Fristedt, R., Willig, A., Granath, P., Crevecoeur, M., Rochaix, J. D., and Vener, A. V. (2009). Phosphorylation of photosystem II controls functional macroscopic folding of photosynthetic membranes in Arabidopsis. Plant Cell 21, 3950-3964. doi: 10.1105/tpc.109.069435

Genty, B., Briantais, J.-M., and Baker, N. R. (1989). The relationship between the quantum yield of photosynthetic electron transport and quenching of chlorophyll fluorescence. Biochim. Biophys. Acta 990, 87-92. doi: 10.1016/S03044165(89)80016-9

Goral, T. K., Johnson, M. P., Brain, A. P., Kirchhoff, H., Ruban, A. V., and Mullineaux, C. W. (2010). Visualizing the mobility and distribution of chlorophyll proteins in higher plant thylakoid membranes: effects of photoinhibition and protein phosphorylation. Plant J. 62, 948-959. doi: 10.1111/j.0960-7412.2010. 04207.x.

Goral, T. K., Johnson, M. P., Duffy, C. D., Brain, A. P., Ruban, A. V., and Mullineaux, C. W. (2011). Light-harvesting antenna composition controls the macrostructure and dynamics of thylakoid membranes in Arabidopsis. Plant J. 69, 289-301. doi: 10.1111/j.1365-313X.2011.04790.x

Gruszecki, W. I., Grudzinski, W., Gospodarek, M., Patyra, M., and Maksymiec, W. (2006). Xanthophyll-induced aggregation of LHCII as a switch between lightharvesting and energy dissipation systems. Biochim. Biophys. Acta 1757, 15041511. doi: 10.1016/j.bbabio.2006.08.002

Haferkamp, S., Haase, W., Pascal, A. A., Van Amerongen, H., and Kirchhoff, H. (2010). Efficient light harvesting by photosystem II requires an optimized protein packing density in Grana thylakoids. J. Biol. Chem. 285, 17020-17028. doi: 10.1074/jbc.M109.077750

Herbstova, M., Tietz, S., Kinzel, C., Turkina, M. V., and Kirchhoff, H. (2012). Architectural switch in plant photosynthetic membranes induced by light stress. Proc. Natl. Acad. Sci. U.S.A. 109, 20130-20135. doi: 10.1073/pnas.1214265109

Horton, P., Ruban, A. V., Rees, D., Pascal, A. A., Noctor, G., and Young, A. J. (1991). Control of the light-harvesting function of chloroplast membranes by aggregation of the LHCII chlorophyll-protein complex. FEBS Lett. 292, 1-4. doi: 10.1016/0014-5793(91)80819-O

Horton, P., Ruban, A. V., and Walters, R. G. (1996). Regulation of light harvesting in green plants. Annu. Rev. Plant Physiol. Plant Mol. Biol. 47, 655-684. doi: 10.1146/annurev.arplant.47.1.655

Ishikawa, Y., Nakatani, E., Henmi, T., Ferjani, A., Harada, Y., Tamura, N., et al. (1999). Turnover of the aggregates and cross-linked products of the D1 protein generated by acceptor-side photoinhibition of photosystem II. Biochim. Biophys. Acta 1413, 147-158. doi: 10.1016/S0005-2728(99)00093-6

Johnson, M. P., Goral, T. K., Duffy, C. D. P., Brain, A. P. R., Mullineaux, C. W., and Ruban, A. V. (2011). Photoprotective energy dissipation involves the reorganization of photosystem II light-harvesting complexes in the grana membranes of spinach chloroplasts. Plant Cell 23, 1468-1479. doi: 10.1105/tpc.110.081646

Kamata, T., Hiramoto, H., Morita, N., Shen, J. R., Mann, N. H., and Yamamoto, Y. (2005). Quality control of Photosystem II: an FtsH protease plays an essential role in the turnover of the reaction center D1 protein in Synechocystis PCC 6803 under heat stress as well as light stress conditions. Photochem. Photobiol. Sci. 4, 983-990. doi: 10.1039/b506068k

Khatoon, M., Inagawa, K., Pospisil, P., Yamashita, A., Yoshioka, M., Lundin, B., et al. (2009). Quality control of photosystem II: thylakoid unstacking is necessary to avoid further damage to the D1 protein and to facilitate D1 degradation under light stress in spinach thylakoids. J. Biol. Chem. 284, 25343-25352. doi: 10.1074/jbc.M109.007740

Kirchhoff, H., Haferkamp, S., Allen, J. F., Epstein, D. B., and Mullineaux, C. W. (2008a). Protein diffusion and macromolecular crowding in thylakoid membranes. Plant Physiol. 146, 1571-1578. doi: 10.1104/pp.107.115170 
Kirchhoff, H., Lenhert, S., Buchel, C., Chi, L., and Nield, J. (2008b). Probing the organization of photosystem II in photosynthetic membranes by atomic force microscopy. Biochemistry 47, 431-440. doi: 10.1021/bi7017877

Kirchhoff, H., Sharpe, R. M., Herbstova, M., Yarbrough, R., and Edwards, G. E. (2013). Differential mobility of pigment-protein complexes in granal and agranal thylakoid membranes of C(3) and C(4) plants. Plant Physiol. 161, 497-507. doi: $10.1104 / p p .112 .207548$

Komayama, K., Khatoon, M., Takenaka, D., Horie, J., Yamashita, A., Yoshioka, M. et al. (2007). Quality control of Photosystem II: cleavage and aggregation of heatdamaged D1 protein in spinach thylakoids. Biochim. Biophys. Acta 1767, 838-846. doi: 10.1016/j.bbabio.2007.05.001

Komenda, J., Barker, M., Kuvikova, S., De Vries, R., Mullineaux, C. W., Tichy, M., et al. (2006). The FtsH protease slr0228 is important for quality control of photosystem II in the thylakoid membrane of Synechocystis sp. PCC 6803. J. Biol. Chem. 281, 1145-1151. doi: 10.1074/jbc.M503852200

Kota, Z., Horvath, L. I., Droppa, M., Horvath, G., Farkas, T., and Pali, T. (2002). Protein assembly and heat stability in developing thylakoid membranes during greening. Proc. Natl. Acad. Sci. U.S.A. 99, 12149-12154. doi: 10.1073/pnas.192463899

Lentz, B. R. (1989). Membrane fluidity as detected by diphenylhexatriene probes. Chem. Phys. Lipids 50, 171-190. doi: 10.1016/0009-3084(89)90049-2

Li, X. P., Bjorkman, O., Shih, C., Grossman, A. R., Rosenquist, M., Jansson, S., et al. (2000). A pigment-binding protein essential for regulation of photosynthetic light harvesting. Nature 403, 391-395. doi: 10.1038/35000131

Lupinkova, L., and Komenda, J. (2004). Oxidative modifications of the Photosystem II D1 protein by reactive oxygen species: from isolated protein to cyanobacterial cells. Photochem. Photobiol. 79, 152-162. doi: 10.1562/00318655(2004)079<0152:OMOTPI>2.0.CO;2

Mori, H., and Yamamoto, Y. (1992). Deletion of antenna chlorophyll-a-binding proteins Cp43 and Cp47 by tris-treatment of Ps-Ii membranes in weak light evidence for a photo-degradative effect on the Ps-Ii components other than the reaction center-binding proteins. Biochim. Biophys. Acta 1100, 293-298. doi 10.1016/0167-4838(92)90484-U

Mori, H., Yamashita, Y., Akasaka, T., and Yamamoto, Y. (1995). Further characterization of the loss of antenna chlorophyll-binding protein $\mathrm{Cp} 43$ from photosystem-I during donor-side photoinhibition. Biochim. Biophys. Acta 1228, 37-42. doi: 10.1016/0005-2728(94)00156-Y

Ohira, S., Morita, N., Suh, H. J., Jung, J., and Yamamoto, Y. (2005). Quality control of photosystem II under light stress - turnover of aggregates of the D1 protein in vivo. Photosynth. Res. 84, 29-33. doi: 10.1007/s11120-004-7310-7

Ruban, A. V., Calkoen, F., Kwa, S. L. S., Vangrondelle, R., Horton, P., and Dekker, J. P. (1997). Characterisation of LHC II in the aggregated state by linear and circular dichroism spectroscopy. Biochim. Biophys. Acta 1321, 61-70. doi: 10.1016/S00052728(97)00047-9

Ruban, A. V., and Horton, P. (1992). Mechanism of delta-Ph-dependent dissipation of absorbed excitation-energy by photosynthetic membranes. 1. Spectroscopic analysis of isolated light-harvesting complexes. Biochim. Biophys. Acta 1102, 30 38. doi: 10.1016/0005-2728(92)90061-6

Sarcina, M., Bouzovitis, N., and Mullineaux, C. W. (2006). Mobilization of photosystem II induced by intense red light in the Cyanobacterium Synechococcus $\mathrm{sp}$ PCC7942. Plant Cell 18, 457-464. doi: 10.1105/tpc.105.035808

Sarcina, M., Murata, N., Tobin, M. J., and Mullineaux, C. W. (2003). Lipid diffusion in the thylakoid membranes of the Cyanobacterium Synechococcus sp.: effect of fatty acid desaturation. FEBS Lett. 553, 295-298. doi: 10.1016/S00145793(03)01031-7

Stoitchkova, K., Busheva, M., Apostolova, E., and Andreeva, A. (2006). Changes in the energy distribution in mutant thylakoid membranes of pea with modified pigment content. II. Changes due to magnesium ions concentration. J. Photochem. Photobiol. B 83, 11-20. doi: 10.1016/j.jphotobiol.2005.11.011
Taiz, L., and Zeiger, E. (2006). Plant Physiology. Sunderland, MA: Sinauer Associates, Inc.

Tardy, F., and Havaux, M. (1997). Thylakoid membrane fluidity and thermostability during the operation of the xanthophyll cycle in higher-plant chloroplasts. Biochim. Biophys. Acta 1330, 179-193. doi: 10.1016/S0005-2736(97) 00168-5

Vener, A. V. (2007). Environmentally modulated phosphorylation and dynamics of proteins in photosynthetic membranes. Biochim. Biophys. Acta 1767, 449-457. doi: 10.1016/j.bbabio.2006.11.007

Wilk, L., Grunwald, M., Liao, P. N., Walla, P. J., and Kuhlbrandt, W. (2013). Direct interaction of the major light-harvesting complex II and PsbS in nonphotochemical quenching. Proc. Natl. Acad. Sci. U.S.A. 110, 5452-5456. doi: 10.1073/pnas.1205561110

Yamamoto, H. Y., and Kamite, L. (1972). The effects of dithiothreitol on violaxanthin de-epoxidation and absorbance changes in the 500-nm region. Biochim. Biophys. Acta 267, 538-543. doi: 10.1016/0005-2728(72)90182-X

Yamamoto, Y. (2001). Quality control of photosystem II. Plant Cell Physiol. 42, 121-128. doi: 10.1093/pcp/pce022

Yamamoto, Y., and Akasaka, T. (1995). Degradation of antenna chlorophyll-binding protein CP43 during photoinhibition of photosystem II. Biochemistry 34, 90389045. doi: 10.1021/bi00028a012

Yamamoto, Y., Aminaka, R., Yoshioka, M., Khatoon, M., Komayama, K., Takenaka, D., et al. (2008). Quality control of photosystem II: impact of light and heat stresses. Photosynth. Res. 98, 589-608. doi: 10.1007/s11120-008-9372-4

Yamamoto, Y., Ford, R. C., and Barber, J. (1981). Relationship between thylakoid membrane fluidity and the functioning of pea chloroplasts : effect of cholesteryl hemisuccinate. Plant Physiol. 67, 1069-1072. doi: 10.1104/pp.67.6. 1069

Yamamoto, Y., Nishi, Y., Yamasaki, H., Uchida, S., and Ohira, S. (2004). Assay of photoinhibition of photosystem II and protease activity. Methods Mol. Biol. 274, 217-227. doi:10.1385/1-59259-799-8:217

Yoshioka, M., Nakayama, Y., Yoshida, M., Ohashi, K., Morita, N., Kobayashi, H., et al. (2010). Quality control of photosystem II: FtsH hexamers are localized near photosystem II at grana for the swift repair of damage. J. Biol. Chem. 285, 41972-41981. doi: 10.1074/jbc.M110.117432

Yoshioka, M., Uchida, S., Mori, H., Komayama, K., Ohira, S., Morita, N., et al. (2006). Quality control of photosystem II - cleavage of reaction center D1 protein in spinach thylakoids by FtsH protease under moderate heat stress. J. Biol. Chem. 281, 21660-21669. doi: 10.1074/jbc.M602896200

Conflict of Interest Statement: The authors declare that the research was conducted in the absence of any commercial or financial relationships that could be construed as a potential conflict of interest.

Received: 24 August 2013; accepted: 11 October 2013; published online: 29 October 2013.

Citation: Yamamoto Y, Hori H, Kai S, Ishikawa T, Ohnishi A, Tsumura $N$ and Morita N (2013) Quality control of Photosystem II: reversible and irreversible protein aggregation decides the fate of Photosystem II under excessive illumination. Front. Plant Sci. 4:433. doi: 10.3389/fpls.2013.00433

This article was submitted to Plant Physiology, a section of the journal Frontiers in Plant Science.

Copyright (C) 2013 Yamamoto, Hori, Kai, Ishikawa, Ohnishi, Tsumura and Morita. This is an open-access article distributed under the terms of the Creative Commons Attribution License (CC BY). The use, distribution or reproduction in other forums is permitted, provided the original author(s) or licensor are credited and that the original publication in this journal is cited, in accordance with accepted academic practice. No use, distribution or reproduction is permitted which does not comply with these terms. 